\title{
IMAGING OF DEEP SINKHOLES USING THE MULTI-ELECTRODE RESISTIVITY IMPLANT TECHNIQUE (MERIT) CASE STUDIES IN FLORIDA
}

\author{
David Harro \\ The G3 Group, 2509 Success Drive, Suite 1, Odessa, FL 33556, david.harro@geo3group.com \\ Henok Kiflu \\ Dept. of Geology, University of South Florida, 4202 E. Fowler Ave., SCA-528, Tampa, FL 33620, \\ hgkiflu@mail.usf.edu
}

\begin{abstract}
Surface geophysical methods have been extensively utilized for sinkhole investigations. While surface geophysical methods can penetrate to depth where sinkhole development occurs the resolution is typically poor. A detailed understanding of deep raveling zones into sinkhole throat through a new and novel geophysical technique was developed by the authors.

The authors performed over 750 sinkhole investigations on residential properties over a five year period of time, in each case geophysical methods of Ground Penetrating Radar (GPR) or Electrical Resistivity (ER) were performed. Over 1500 confirmatory Standard Penetration Test (SPT) drillings were performed of the geophysical anomalies. In a very large percentage of the geophysical surveys performed, the location, size and depth of the raveling zones into the sinkhole throat could be clearly identified.
\end{abstract}

The authors developed a novel geophysical technique called The Multi-Electrode Resistivity Implant (MERIT) to address the need to image deeper into karst formations to help identify the location of deep raveling zones and sinkhole throats. The purpose of this paper is to present case studies of the application of MERIT technology. Three case studies are presented in this paper.

The first case study focuses on the first application of MERIT at the Bordeaux Village in Tampa, Florida where a sinkhole swallowed a car in 2010. The MERIT survey was able to image the car in the sinkhole throat. This case study demonstrates the ability of the MERIT technique to identify the location of the sinkhole throat by identifying the depth and location of the car, a large conductive ER anomaly.

The second case study focuses on a pipeline in Orlando, Florida being threatened by sinkhole development on the adjacent property. MERIT was able to identify size and depth of raveling of soils into the sinkhole throat near the pipeline. The results of the MERIT image were critical in engineering design to address the treatment to the pipeline.

In the third case study MERIT technology was applied to a proposed roadway through an extensive karst region of Lake County, Florida. Initial geotechnical investigation indicated a potentially large and deep sinkhole feature. MERIT was able to provide a concise geologic structure including the identification of the locations sinkhole throat at $52 \mathrm{~m}$ deep.

MERIT has been shown to identify details of the complex geology and geometry of karst formations. In particular the techniques ability to provide improved image capabilities of the raveling zone and sinkhole throats has significant engineering applications for assessment, risk analysis, and remediation of sinkholes.

\section{Introduction}

Multi-Electrode Resistivity Implant Technique (MERIT) is a technique that utilizes a tomographic configuration (Figure 1) that combines measurements with surface and deep electrodes that improve geophysical surveys using electrical resistivity (Harro \& Kruse, 2013).

The tomographic arrangement of electrical resistivity data required new algorithms the development of new optimal array geometries and extensive laboratory and field research (Loke et al., 2015). The buried arrays are identical in length and electrode spacing as the surface array creating a mirror image in the subsurface. The tomographic configuration creates a vertical stacking of the electrical resistivity data resulting in the ability to reduce the survey length, increase penetration and increase image resolution at depth. MERIT has been shown to significantly improve resolution over 


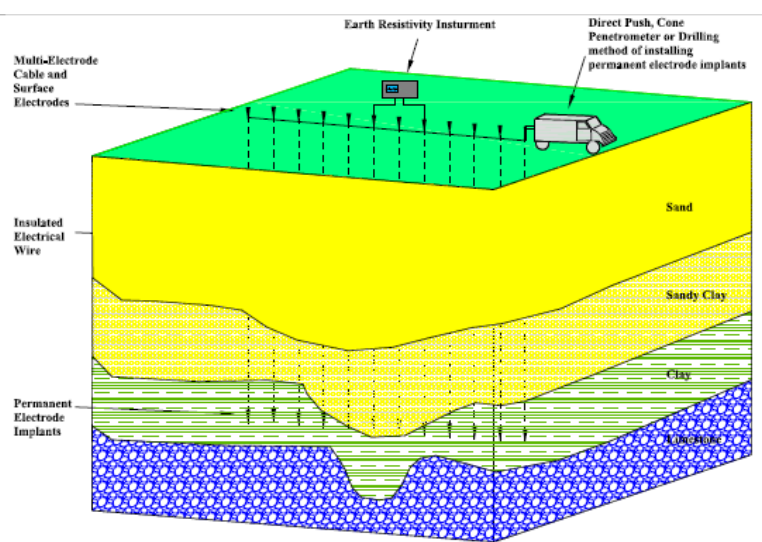

Figure 1. Deployment of implants for MERIT, note tomographic configuration of identical spaced surface and lower arrays.

basic arrays adapted from traditional 2D ER surface geometries (Kiflu et al., 2016).

Three case studies are used to illustrate the benefits of the MERIT technique. While all case studies represent cover-collapse conditions in west central Florida, each case study has some unique conditions that include surface limitations, buried utilities/infrastructure and changes in depth of targets. These case studies include:

- Revisit to the Bordeaux Village apartment complex in Tampa, Florida where the full scale trial of the MERIT technique was deployed. Measurements were repeated in the same area with improved array geometries and processing algorithms.

- A large sinkhole and area of subsidence developed on residential property on Salmon Drive in Orlando, Florida. The area of subsidence covered a $60 \mathrm{~m}$ radius that extended off the property toward the Florida Turnpike impacting the sound barrier and two lanes of the highway.

- A relic sinkhole in Lake County, Florida along a proposed Wekiva parkway

\section{Case Study - Revisit Bordeaux Apartments Tampa, Florida}

The Bordeaux Village apartments in Tampa, Florida received national news coverage in July 2010 after a car in the parking lot was swallowed by a $6 \mathrm{~m}$ diameter cover collapse sinkhole (Figure 2).

The MERIT first full scale field trial was performed to help identify the potential geometry of the sinkhole in

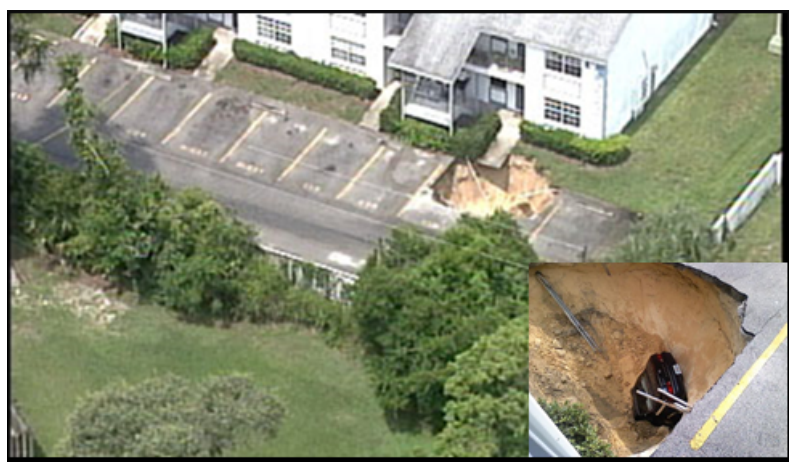

Figure 2. Bordeaux Apartments sinkhole, Tampa, FL, aerial photo showing car being shallowed by a sinkhole.

2012. The Bordeaux Village apartments were revisited with improved array geometries and algorithms.

The second set of data was acquired using the original MERIT survey configuration that comprised 18 surface electrodes and 18 implant locations at $3 \mathrm{~m}$ spacing adjacent to the location of the sinkhole. The MERIT implants were positioned at a depth of $9 \mathrm{~m}$ and were in contact with Hawthorn Formation clays and clayey sands and were within $3 \mathrm{~m}$ of the average depth of the top of rock (Harro \& Kruse, 2013).

The MERIT survey data were collected using an AGI super sting resistivity instrument and was inverted using Res2Dinv software from Geotomo, Inc. The MERIT image shown in the Figure 4 profile details an upper sand unit that is resistive (red) that overlies conductive Hawthorne Formation sandy clay grading to clay that is conductive (blue), which in turn overlies the resistive (red) limestone formation. From the MERIT image vertical variations in limestone layers as well as the sinkhole throat can be seen.

The original investigation included two SPT and their locations are shown in Figure 3. The SPT encountered fine sand to $4 \mathrm{~m}$, after which sandy clay to clay ranging in thickness from 3 to $6 \mathrm{~m}$ thick was recorded overlying the top of the limestone at depths between $10 \mathrm{~m}$ and $13 \mathrm{~m}$ with competent limestone starting at $15 \mathrm{~m}$.

In the MERIT image the upper resistive unit (sand) has an average depth of $4 \mathrm{~m}$. The conductive unit (sandy clay to clay) below this has an average thickness of $6 \mathrm{~m}$. The top limestone (resistive) in the MERIT image can be seen at an average depth of $12 \mathrm{~m}$. 


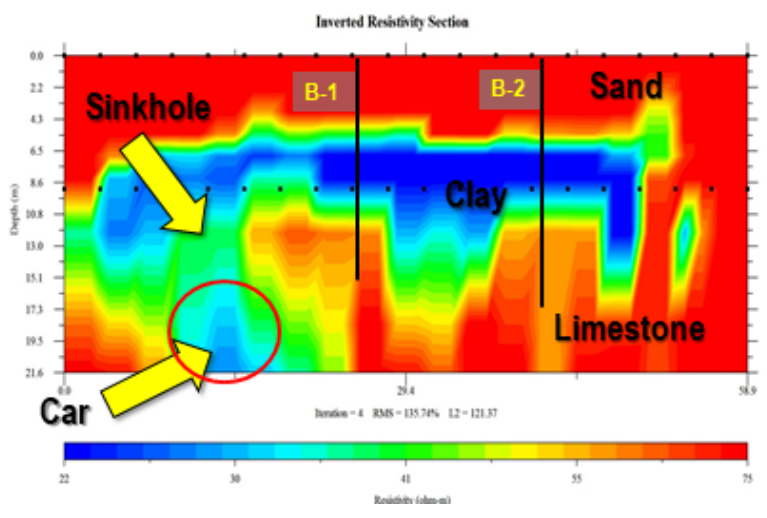

Figure 3. MERIT image showing a highly conductive anomaly inside the sinkhole throat. The location of the sinkhole and car was verified by measurements taken during the initial sinkhole collapse.

The depth position of the car was measured before it disappeared into the sinkhole. The car was vertically positioned, measuring $4 \mathrm{~m}$ in length and was last seen at a depth of $6 \mathrm{~m}$ in the sinkhole. In the MERIT image a highly conductive anomaly appears at 17 to 21 meters inside the sinkhole throat. The conductive anomaly is aligned with the last known position of the car.

\section{Case Study Sinkhole Florida Turnpike}

A large sinkhole developed on a residential property located on Salmon Drive in Orlando, Florida. The sinkhole feature was located on the eastern side of the residential property adjacent to the Florida Turnpike (Figure 4).

After the sinkhole development, a $60 \mathrm{~m}$ radius affected area extended from the sinkhole on the residential property and impacted two of the southbound lanes of the Florida Turnpike. Significant signs of ground subsidence included slumping of the two lanes and up to $15 \mathrm{~cm}$ of differential movement of the sound barrier. A section of a pressurized reclaimed water transmission main is located in the area of ground subsidence.

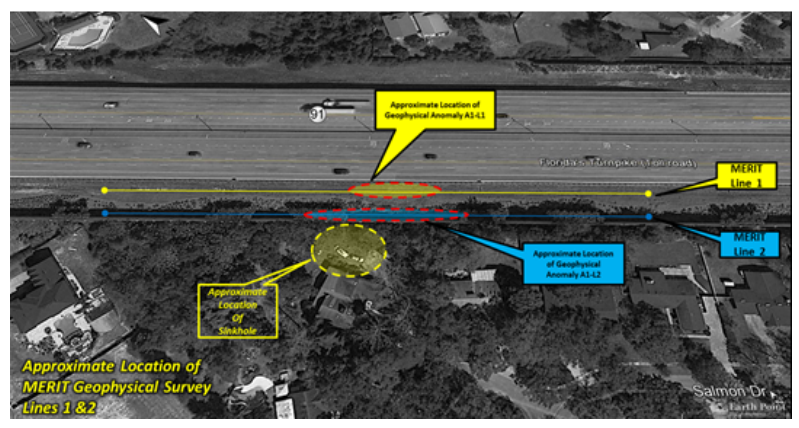

Figure 4. Location of the MERIT survey, pipeline, and sinkhole on the adjacent property.
Concerns that the sinkhole could pose a potential risk to the pipeline prompted the geotechnical investigation, and a geophysical survey using MERIT was requested. The MERIT geophysical survey consisted of 28 surface and 28 implanted electrodes over $165 \mathrm{~m}$ (540 feet). Implants were positioned at a depth of $15 \mathrm{~m}$ (50 feet) in order to obtain a depth of $45 \mathrm{~m}$ (150 feet).

The results of the MERIT geophysical survey of Line 1 identified a distinct geophysical anomaly located in the subsurface between $54 \mathrm{~m}$ (180 feet) and $85 \mathrm{~m}$ (280 feet) (Figure 5). The anomaly was located within the area of the highest concentration of distress/ground subsidence observed on the roadway and the sound barriers as well as being adjacent to the corresponding sinkhole development on the adjacent property.

The geophysical anomaly identified suggests that the sand unit has in the past or has recently moved downward and laterally into the underlying clay unit in the direction of the sinkhole. This would correspond with the sinkhole development type called cover-collapse. Cover-collapse sinkhole formation occurs when the underlying limestone is covered by a significant layer of clay. Dissolution of the limestone creates a void in the clay which will eventually collapse. If the clay has significant amounts of sand material covering it, the sand will infiltrate the voids created.

Three SPT borings were performed by the geotechnical consultant along a profile in the affected area prior to the implementation of the MERIT survey. SPT borings encountered sands grading to more silty sands to a depth of $24 \mathrm{~m}$ (78 feet) transitioning to sandy silty clay to silty clay to depths of $40 \mathrm{~m}$ (130 feet). No limestone was encountered in the borings.

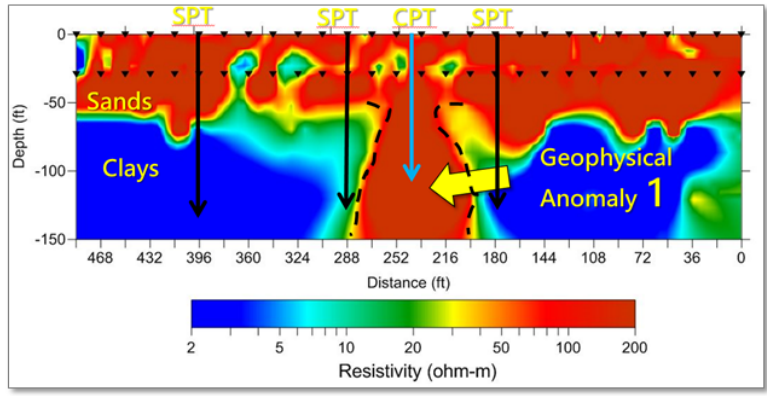

Figure 5. MERIT image shows vertical raveling of sands downward into clay unit. SPT were performed before the MERIT image and the CPT was performed after. 
The results of MERIT shows the initial SPT borings did not intersect the area of concern. After the MERIT survey was performed it was determined a CPT should be performed in the anomalous area. The results of the CPT did indicate loose soils but not direct sinkhole conditions. Therefore based on the location of testing and the distress to the surrounding area, the MERIT geophysical anomaly most likely represented lateral movement of the upper sand unit into the sinkhole to the west.

Additional MERIT Line 2 was performed along the sound barrier adjacent to the sinkhole. The results of the MERIT Line 2 could not be verified by drilling due to physical constraints.

Based on the results of MERIT the risk to the pipeline was evaluated as to be enough potential to redesign the pipe line by constructing a bridge for the pipeline over the length of area of $21 \mathrm{~m}$ identified by the MERIT geophysical survey.

\section{Case Study Wekiva Parkway CR46A}

A new roadway was to be established by the Florida Department of Transportation (FDOT) in Lake County, Florida called CR46A or the Wekiva Parkway. The area of the road is well known to have karst geology. A review of aerial photographs along the alignment identified several potential karst features. These were investigated by the FDOT geotechnical consultant. After the initial SPT boring was completed in the area (referred to as site B) it was determined the potential large relic sinkhole was present (Figure 6).

A preliminary geotechnical investigation was conducted by others at Site B which included: GPR and in total the drilling of 26 SPTs and CPTs to better define the depth and extent of the relic sinkhole. SPT boring R-1 performed in the alignment encountered loose to medium dense fine sand to fine sand with silt to a depths of $8.5 \mathrm{~m}$ (28 feet); followed by a layer of loose to medium dense mucky fine sand to a depth of $13 \mathrm{~m}$ (43 feet); underlain by very loose to loose silty sand to clayey sand to a depth of $25 \mathrm{~m}$ (85 feet); followed by medium dense to very dense limestone to the boring termination depth of $45 \mathrm{~m}$ (150 feet) below existing ground surface. Drilling fluid circulation loss occurred at depths between $21 \mathrm{~m}$ (70 feet) and $41 \mathrm{~m}$ (135 feet).

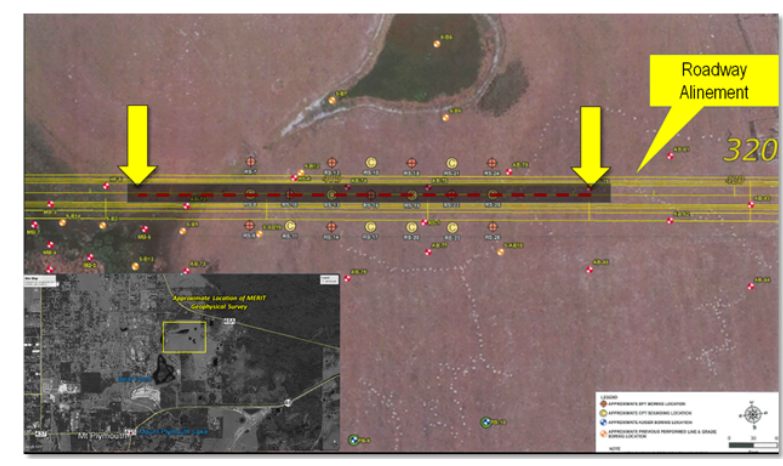

Figure 6. Google Earth image showing the alignment of new roadway CR46A and the location of the MERIT geophysical survey as well as SPTs and CPTS.

The MERIT geophysical survey was recommended after the geotechnical results indicated the size of the depth of the relic sinkhole required greater understanding.

Based on the geotechnical investigation performed by a consultant and the FDOT, the limestone formation was encountered at depths between $18 \mathrm{~m}$ and $45 \mathrm{~m}$. The high degree of variability of the limestone formation encountered in the geotechnical investigation identified the potential for karst or sinkhole conditions that are considered to be a concern for this project. The application of the MERIT techniques' abilities to provide deep geophysical images was deemed beneficial to the project.

A complete profile across the relic sinkhole was performed using 28 surface electrodes and 28 implants over a $165 \mathrm{~m}$ linear distance at $6 \mathrm{~m}$ spacing. The implants were installed to an average depth of $14 \mathrm{~m}$ bls. Based on the subsurface geometry, MERIT data collection took over 5 hours to complete and encompassed over 3000 data points (Figure 7).

The MERIT survey of the sinkhole clearly identified the sinkhole throat of $52 \mathrm{~m}$ with a span of $45 \mathrm{~m}$. The throat can be seen along the profile length between 120 and 300 foot intervals. Adjacent to the sinkhole throat is highly resistive and competent limestone Stratum 4 (red) of varying geometry along the profile between 0 to $37 \mathrm{~m}$ and on the other side of the sinkhole throat at $91 \mathrm{~m}$ to $152 \mathrm{~m}$. A large section of weathered limestone (Stratum 3) can be seen as the greenish area between $85 \mathrm{~m}$ and $165 \mathrm{~m}$ extending upward to nearly $15 \mathrm{~m}$. Of note is where CPT was performed to $45 \mathrm{~m}$ at profile distance $134 \mathrm{~m}$, through a void in Stratum 3 material. Stratum 2 is comprised of 


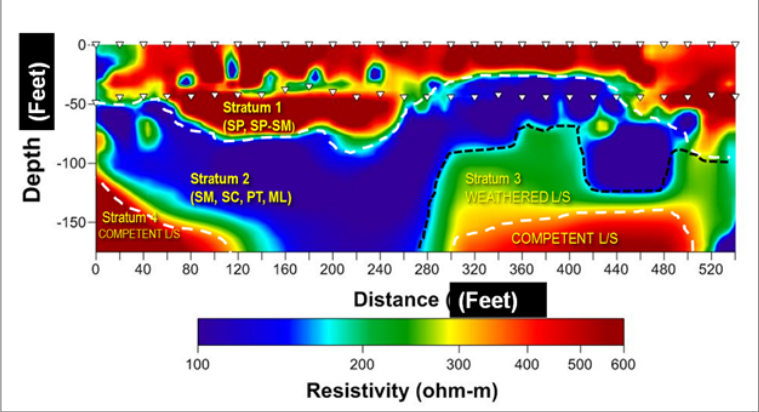

Figure 7. MERIT image showing geometry, and the sinkhole throat at 170 as well as a depressive area in the upper (resistive) sand unit into the conductive unit of material that infilled the sinkhole.

conductive material silts, clays, and organics that are associated with material that infilled the sinkhole after initial collapse. This conductive material provides a sharp contrast to the resistive sand and limestone to help define the geometry of the sinkhole. Overlying are the highly resistive sands of Stratum 1. Of special note is the depression located in Stratum 1 between $15 \mathrm{~m}$ and $73 \mathrm{~m}$ along the profile. This suggests a reactivation of the sinkhole resulting in the Stratum 2 material that was filled in by sands of Stratum 1. CPT testing in the area of the depression clearly showed a significant reduction in tip pressure located at the Stratum 1/ Stratum 2 boundary, and the SPT confirmed the thickness and boundary of the depression as well.

The results of the MERIT profile indicated a very good correlation with SPT and CPT data obtained during the geotechnical investigation. A comparison of deep SPT borings indicated similar depths of all stratums as the MERIT profile.

The CPT results taken along the center line were compared with the results of MERIT along the primary line. There is a good correlation between the MERIT Stratum boundaries and the results of the CPT's soil behavior type and noticeable changes in tip resistance.

\section{Conclusion}

The case studies presented here are intended to provide a general understanding and view of the potential of implant technology for geophysical surveys, especially in regions of karst. While the MERIT technique is minimally invasive and requires more investment in time and cost, the results are significantly improved over surface geophysical methods. The ability to clearly identify sinkhole geometry, geology, raveling zones and potentially the locations of the throat at depths of over $30 \mathrm{~m}$ (100 feet) can lead to much greater understanding or engineering applications of risk analysis, monitoring and remediation of sinkholes. If we are to gain a greater understanding of the enigmatic geology we call "karst", implant technology such as MERIT can lead the way to increase our understanding which will result in better decision-making.

\section{References}

Harro D, Kruse S. 2013. Improved imaging of covered karst with the multi-electrode resistivity implant technique. NCKRI Symposium 2. Proceedings of the 13th Multidisciplinary Conference on Sinkholes and the Engineering and Environmental Impacts of Karst. Carlsbad, New Mexico, US.

Loke MH, Kiflu H, Wilkinson P, Harro D, Kruse S. 2015. Optimized arrays for 2-D resistivity surveys with combined surface and deep arrays. Near Surface Geophysics, 13, 505-517.

Kiflu H, Kruse S, Loke, MH, Wilkinson P, Harro D. 2016. Improving resistivity survey resolution at sites with limited spatial extent using buried electrode arrays. Journal of Applied Geophysics, 135: 338-355. 
